\title{
MINERALIZAÇÃO POTENCIAL E LÍQUIDA DE NITROGÊNIO EM SOLOS ${ }^{(1)}$
}

\author{
Renato Yagi ${ }^{(2)}$, Manoel Evaristo Ferreira ${ }^{(3)}$, Mara Cristina Pessôa \\ da Cruz ${ }^{(4)}$ \& José Carlos Barbosa ${ }^{(5)}$
}

\begin{abstract}
RESUMO
Os métodos de incubação de solo fornecem subsídios técnicos para a avaliação da mineralização e disponibilidade de $\mathrm{N}$. Nesse sentido, os objetivos deste trabalho foram avaliar a mineralização potencial e líquida do $\mathrm{N}$ orgânico em solos e relacioná-la à disponibilidade de $\mathrm{N}$ para plantas. Amostras de 22 solos coletadas na camada arável $(0 \mathrm{a} 20 \mathrm{~cm})$ foram submetidas à incubação em condições aeróbias a $35{ }^{\circ} \mathrm{C}$, por 30 semanas, e anaeróbias a $40{ }^{\circ} \mathrm{C}$, por sete dias, e utilizadas em experimento, em casa de vegetação, em que o milho foi a planta-teste. Durante a incubação por 30 semanas, houve aumento do $\mathrm{N}$ mineralizado na segunda semana, seguido de diminuição e estabilização a partir da quarta semana. Este comportamento resultou em ajuste do $\mathrm{N}$ mineralizado $\left(\mathrm{N}_{\mathrm{m}}\right)$ ao modelo exponencial de decrescimento e do $\mathrm{N}$ mineralizado acumulado $\left(\mathrm{N}_{\mathrm{mac}}\right)$ ao modelo exponencial de crescimento, a partir do qual foi possível calcular o $\mathrm{N}$ potencialmente mineralizável $\left(\mathrm{N}_{0}\right)$. As quantidades de $\mathrm{N}_{0}$ refletiram a mineralização e a disponibilidade de $\mathrm{N}$ em longo prazo. Contudo, $\mathrm{o} \mathrm{N}_{\text {mac }}$ apresentou maior grau de correlação com o $\mathrm{N}$ disponível medido pelas plantas (concentração de $\mathrm{N}$ e $\mathrm{N}$ acumulado na parte aérea) do que o $\mathrm{N}_{0}$, e valores altos de correlação foram obtidos já após duas semanas de incubação, o que indica que a modelagem matemática pode ser dispensada e o período de incubação pode ser encurtado. $\mathrm{O} N$ total do solo foi melhor índice da mineralização potencial e líquida de $\mathrm{N}$ orgânico do que a matéria orgânica do solo, principalmente em longo prazo. $O$ método de incubação anaeróbia de curta duração pode ser utilizado para cálculo da estimativa do $\mathrm{N}_{0}$, e a subtração do $\mathrm{N}^{-\mathrm{NH}_{4}}{ }^{+}$"inicial” não contribuiu para melhorar a estimativa da mineralização e da disponibilidade de $\mathrm{N}$ por meio deste método, o que o torna ainda mais simples e viável.
\end{abstract}

Termos de indexação: $\mathrm{N}$ potencialmente mineralizável, matéria orgânica do solo, milho.

\footnotetext{
(1) Parte da tese de doutorado do primeiro autor. Financiado pela FAPESP. Recebido para publicação em junho de 2008 e aprovado em fevereiro de 2009.

${ }^{(2)}$ Doutorando em Agronomia (Produção Vegetal), Universidade Estadual Paulista, Faculdade de Ciências Agrárias e Veterinárias - UNESP/FCAV. Via de Acesso Prof. Paulo Donato Castellane s/n, CEP 14884-900 Jaboticabal (SP). Bolsista da FAPESP. E-mail: renatoyagi@yahoo.com.br

${ }^{(3)}$ Professor Titular, Departamento de Solos e Adubos, UNESP/FCAV. E-mail: evaristo@fcav.unesp.br

(4) Professora Assistente Doutora, Departamento de Solos e Adubos, UNESP/FCAV. E-mail: mcpcruz@fcav.unesp.br

(5) Professor Titular, Departamento de Ciências Exatas, UNESP/FCAV. E-mail: jcbarbosa@fcav.unesp.br
} 


\title{
SUMMARY: POTENTIAL AND NET NITROGEN MINERALIZATION IN SOILS
}

\begin{abstract}
The soil incubation methods can be used as tools to a better evaluation of $N$ mineralization and availability. The objective of this study was to evaluate the potential and net soil organic $N$ mineralization, and relate them to $N$ availability to plants. Twenty two soil samples $(0-20 \mathrm{~cm})$ were incubated at $35{ }^{\circ} \mathrm{C}$ for 30 weeks in aerobic conditions and at $40{ }^{\circ} \mathrm{C}$ for seven days in anaerobic conditions, and tested in a greenhouse experiment on corn plants. During the 30 weeks-incubation period $N$ mineralization increased in the $2^{\text {nd }}$ week, followed by a decrease and stabilization from the $4^{\text {th }}$ week afterwards. The mineralized $N$ data was adjusted to an exponential decay model and the accumulated $N$ mineralized $\left(N_{\text {mac }}\right)$ data was adjusted to an exponential growth model and the potentially mineralizable $N\left(N_{0}\right)$ was then estimated. The quantities of $N_{0}$ expressed $N$ mineralization and availability in the long-term. However, the correlation of $N_{\text {mac }}$ with available $N$ indicated by plants ( $N$ concentration and $N$ uptake) was higher than of $N_{0}$, and high correlation values were found after two weeks of incubation. This indicates that the statistical adjustment of data may be unnecessary and the incubation period can be reduced. The soil total $N$ was a better indicator of the net and potential $N$ organic mineralization than soil organic matter, especially in the long-term. The incubation for seven days in anaerobic conditions can be used to estimate $\mathrm{N}_{o}$. The subtraction of initial $\mathrm{N}-\mathrm{NH}_{4}{ }^{+}$ from the data obtained after incubation did not improve the estimation of $N$ mineralization and availability by this method, which makes it even more simple and feasible.
\end{abstract}

Index terms: potentially mineralizable $N$, soil organic matter, corn.

\section{INTRODUÇÃO}

A mineralização mais rápida do $\mathrm{N}$ orgânico adicionado ao solo tem sido atribuída mais à labilidade e recalcitrância do que especificamente à estrutura dos compostos, uma vez que as formas recémadicionadas de $\mathrm{N}$ orgânico e as recém-imobilizadas são rapidamente convertidas nas formas presentes na matéria orgânica do solo (Zech et al., 1997). Além do caráter lábil ou recalcitrante do $\mathrm{N}$ orgânico, outros fatores como condições edáficas e climáticas e atividade microbiana alteram a capacidade intrínseca de cada solo de fornecer $\mathrm{N}$ inorgânico para as plantas (Camargo et al., 1997; Rhoden et al., 2006). Condições edáficas como textura, estrutura, temperatura, umidade, acidez/alcalinidade, fertilidade do solo interferem na cinética de mineralização do N orgânico (Zech et al., 1997; Gonçalves et al., 2001).

Para medir a capacidade de mineralização do $\mathrm{N}$ orgânico são utilizados métodos de incubação de amostras de solo em temperatura, umidade e disponibilidade de nutrientes padronizadas, nos quais a liberação do N inorgânico é determinada em extratos obtidos por percolação ou agitação de amostras com solução salina diluída (Wang et al., 2003). O potencial de mineralização líquida do $\mathrm{N}$ orgânico dos solos é estimado mediante ajuste a modelos matemáticos, que, por sua vez, são utilizados para interpretar a resposta microbiana (Camargo et al., 1997). A inclusão de diversas variáveis nos modelos matemáticos resulta em melhora nos ajustes, mas dificulta a compreensão do seu significado biológico (Wang et al., 2003). Neste caso, não são raros os trabalhos que utilizam modelagem matemática complexa, mas que não exploram a utilização prática e agronômica dos resultados.
Tradicionalmente, o modelo matemático mais estudado é o exponencial de crescimento, também conhecido como modelo exponencial simples, que foi proposto por Stanford \& Smith (1972) para ajuste dos resultados obtidos com o método de incubação aeróbia de solo de longa duração. O modelo pressupõe que a taxa de mineralização do N orgânico seja proporcional ao substrato mineralizável do solo e que tal fenômeno seja explicado pela equação: $\mathrm{N}_{\mathrm{mac}}=\mathrm{N}_{0}\left(1-\mathrm{e}^{-\mathrm{kt}}\right)$, em que $\mathrm{N}_{\text {mac }}=\mathrm{N}$ mineralizado acumulado; $\mathrm{N}_{0}=\mathrm{N}$ potencialmente mineralizável; $\mathrm{k}=$ constante da taxa de mineralização e $t=$ tempo. Os valores de $\mathrm{N}_{0}$ dos solos têm sido utilizados como padrão de referência em trabalhos de seleção de métodos químicos para a estimativa do $\mathrm{N}$ orgânico mineralizável do solo (Gianello \& Bremner, 1986; Curtin \& Wen, 1999). De acordo com Gonçalves et al. (2001), de posse dos valores de $\mathrm{N}_{0}$, de $\mathrm{k}$ e das informações das exigências nutricionais de determinada cultura, podem-se também estimar o estoque de $\mathrm{N}$ para as plantas e prever a resposta à adubação nitrogenada. Além desse modelo matemático, outros têm sido estudados, como os modelos exponenciais duplos, que supõem que existem duas frações de $\mathrm{N}$ orgânico mineralizável (Camargo et al., 2002).

Métodos de incubação de solo de curta duração também são utilizados como índices de disponibilidade de $\mathrm{N}$ para as plantas e como padrões de referência para métodos químicos (Keeney \& Bremner, 1966; Gianello \& Bremner, 1986; Cantarella et al., 1994), e são mais simples, rápidos e com custos menores que os de longa duração (Boeira \& Maximiliano, 2004). Diante dessas vantagens e da correlação $\left(R=0,92^{* *}\right)$ observada entre as quantidades de N mineralizadas em solos que receberam doses de lodo de esgoto e foram 
submetidos à incubação de longa e curta durações, os últimos autores propuseram o método de incubação de curta duração em condições anaeróbias para a estimativa da fração de mineralização de N orgânico de lodos de esgoto. Tanto os resultados dos métodos de curta duração quanto os daqueles de longa duração têm apresentado correlações significativas com o N absorvido por plantas-teste (Keeney \& Bremner, 1966; Cantarella et al., 1994; Camargo et al., 1997; Rhoden et al., 2006).

Um dos métodos de incubação de solo de curta duração mais utilizados é o desenvolvido em condições anaeróbias (watterlogged), proposto por Waring \& Bremner (1964) e adaptado por Keeney \& Bremner (1966) para reduzir o tempo de incubação de 14 para sete dias, com aumento de temperatura de 30 para $40{ }^{\circ} \mathrm{C}$. Nesse método, os autores recomendam subtrair das quantidades de $\mathrm{N}-\mathrm{NH}_{4}{ }^{+}$mineralizadas o N$\mathrm{NH}_{4}{ }^{+}$"inicial" do solo obtido por meio de destilação da suspensão de solo com solução de KCl. Sahrawat \& Ponnamperuma (1978) verificaram que, com a destilação das suspensões de oito solos das Filipinas, foram extraídos, em média, $33 \mathrm{mg} \mathrm{dm}^{-3} \mathrm{de} \mathrm{N} \mathrm{NH}_{4}{ }^{+}$a mais do que com a destilação dos extratos filtrados. Segundo esses autores, a destilação de suspensões de solo com $\mathrm{KCl}$ e $\mathrm{MgO}$ resulta em hidrólise da matéria orgânica do solo em valores altos de $\mathrm{pH}$, ocasionando erros na medida do $\mathrm{N}-\mathrm{NH}_{4}{ }^{+}$em solos tropicais. Por outro lado, a não-subtração das quantidades de $\mathrm{N}-\mathrm{NH}_{4}{ }^{+}$ mineralizadas durante a incubação anaeróbia pode representar melhor a mineralização e a disponibilidade de $\mathrm{N}$ para as plantas.

Pelo exposto, os objetivos deste trabalho foram avaliar a mineralização potencial e líquida de $\mathrm{N}$ orgânico de solos e relacioná-la à disponibilidade de $\mathrm{N}$ para plantas de milho.

\section{MATERIAL E MÉTODOS}

O trabalho foi realizado na Unesp/FCAV, Campus de Jaboticabal (SP), empregando 22 solos do planalto ocidental do Estado de São Paulo. A cobertura vegetal e a classificação dos solos de acordo com Embrapa (1999) estão no quadro 1. As amostras foram coletadas na camada de 0 a $20 \mathrm{~cm}$, espalhadas sobre plástico em camadas de no máximo $10 \mathrm{~cm}$ de espessura, e revolvidas periodicamente para secagem rápida e interrupção da atividade microbiana. Depois de secas, as amostras foram destorroadas, passadas por peneira de $4 \mathrm{~mm}$ de abertura de malha, homogeneizadas e amostradas para caracterização granulométrica (Camargo et al., 1986), determinação de pH, matéria orgânica, bases trocáveis, acidez total (Raij et al., 2001) e N total (Tedesco et al., 1985) (Quadro 1). As amostras tomadas para caracterização granulométrica e química (exceto $\mathrm{N}$ total) foram passadas por peneira

Quadro 1. Solos submetidos às incubações aeróbia e anaeróbia e utilizados no experimento em casa de vegetação

\begin{tabular}{|c|c|c|c|c|c|c|c|c|c|}
\hline Solo & Sigla ${ }^{(1)}$ & $\begin{array}{l}\text { Municipio } \\
\text { de coleta }\end{array}$ & Vegetação & Areia & Argila & N total & $\operatorname{MOS}^{(2)}$ & $\mathrm{pH} \mathrm{CaCl}_{2}$ & $\mathbf{V}$ \\
\hline & & & & & $\mathrm{g} \mathrm{kg}^{-1}$ & - & $\mathrm{g} \mathrm{dm}^{-3}$ & & $\%$ \\
\hline 1 & LV1 & Jaboticabal & Seringueira & 600 & 370 & 0,93 & 24 & 4,4 & 26 \\
\hline 2 & $\mathrm{LV} 2$ & Jaboticabal & Eucalipto & 570 & 380 & 0,96 & 35 & 4,0 & 16 \\
\hline 3 & LV3 & Jaboticabal & Pinheiro & 590 & 370 & 0,79 & 25 & 5,0 & 53 \\
\hline 4 & PVA1 & Monte Alto & Eucalipto & 860 & 100 & 0,59 & 21 & 5,0 & 47 \\
\hline 5 & LV4 & Jaboticabal & Florestais nativas & 240 & 510 & 2,82 & 60 & 6,2 & 88 \\
\hline 6 & PVA2 & Pindorama & Seringueira & 870 & 70 & 0,82 & 26 & 5,7 & 64 \\
\hline 7 & PVA3 & Pindorama & Goiabeira & 850 & 80 & 0,70 & 24 & 5,3 & 60 \\
\hline 8 & PVA4 & Pindorama & Florestais nativas & 740 & 210 & 1,41 & 34 & 5,0 & 51 \\
\hline 9 & PVA5 & Pindorama & Cana-de-açúcar & 850 & 100 & 0,50 & 17 & 5,5 & 67 \\
\hline 10 & LV5 & Jaboticabal & Soja & 500 & 430 & 0,93 & 24 & 5,8 & 69 \\
\hline 11 & LV6 & Ribeirão Preto & Seringueira & 170 & 430 & 1,82 & 60 & 5,2 & 55 \\
\hline 12 & LV7 & Ribeirão Preto & Seringueira & 110 & 570 & 2,25 & 61 & 4,8 & 42 \\
\hline 13 & LV8 & Ribeirão Preto & Eucalipto & 120 & 590 & 2,07 & 55 & 4,6 & 34 \\
\hline 14 & LV9 & Jaboticabal & Milho & 570 & 390 & 0,83 & 30 & 5,0 & 47 \\
\hline 15 & LV10 & Jaboticabal & Florestais nativas & 600 & 320 & 1,02 & 35 & 5,0 & 53 \\
\hline 16 & LV11 & Sertãozinho & Capim braquiária & 110 & 510 & 2,15 & 56 & 5,1 & 50 \\
\hline 17 & LV12 & Sertãozinho & Florestais nativas & 220 & 560 & 2,34 & 54 & 4,7 & 45 \\
\hline 18 & $\mathrm{LV} 13$ & Jaboticabal & Florestais nativas & 650 & 330 & 0,77 & 23 & 4,0 & 6 \\
\hline 19 & $\mathrm{LV} 14$ & Jaboticabal & Eucalipto & 690 & 290 & 0,91 & 34 & 4,2 & 18 \\
\hline 20 & NQ & Luís Antônio & Florestais nativas & 870 & 110 & 0,82 & 44 & 4,0 & 5 \\
\hline 21 & $\mathrm{LV} 15$ & Taquaritinga & Pastagem & 820 & 140 & 0,59 & 15 & 5,1 & 50 \\
\hline 22 & LV16 & Taquaritinga & Mangueira & 760 & 210 & 0,54 & 15 & 5,3 & 52 \\
\hline
\end{tabular}

(1) LV: Latossolo Vermelho; PA: Argissolo Vermelho-Amarelo; NQ: Neossolo Quartzarênico. ${ }^{(2)}$ Matéria orgânica do solo. 
de $2 \mathrm{~mm}$ e a amostra para determinação de $\mathrm{N}$ total foi triturada em almofariz e passada por peneira de $0,25 \mathrm{~mm}$.

As amostras dos 22 solos foram submetidas à incubação em condições aeróbias e anaeróbias e utilizadas em experimento em casa de vegetação. Para o método de incubação aeróbia de longa duração, seguiu-se procedimento proposto por Stanford \& Smith (1972), utilizando três repetições. Mistura de solo (15 g) e areia lavada ( $15 \mathrm{~g}$ ) foi colocada entre camadas de lã-de-vidro de $30 \mathrm{~mm}$ e $10 \mathrm{~mm}$, inferior e superior, respectivamente, em tubo percolador com capacidade para $100 \mathrm{~mL}$. No início e após $2,4,8,12,16,22$ e 30 semanas de incubação, a $35^{\circ} \mathrm{C}$, em câmara do tipo "BOD", foram feitas percolações de solução de $\mathrm{CaCl}_{2}$ $0,01 \mathrm{~mol} \mathrm{~L}^{-1}$, determinação de $\mathrm{N}$ mineralizado (N$\mathrm{NH}_{4}{ }^{+}+\mathrm{N}-\mathrm{NO}_{3}{ }^{-}$) nos extratos percolados (Cantarella \& Trivelin, 2001), reposição de solução nutritiva sem $\mathrm{N}\left[\mathrm{CaSO}_{4} \cdot 2 \mathrm{H}_{2} \mathrm{O} 0,002 \mathrm{~mol} \mathrm{~L}^{-1} ; \mathrm{MgSO}_{4} 7 \mathrm{H}_{2} \mathrm{O}\right.$ $0,002 \mathrm{~mol} \mathrm{~L}^{-1} ; \mathrm{Ca}\left(\mathrm{H}_{2} \mathrm{PO}_{4}\right)_{2} \cdot \mathrm{H}_{2} \mathrm{O} 0,005 \mathrm{~mol} \mathrm{~L}^{-1} \mathrm{e} \mathrm{K}_{2} \mathrm{SO}_{4}$ $0,0025 \mathrm{~mol} \mathrm{~L}^{-1}$ e ajuste da umidade do sistema por meio de bomba de vácuo. Para o ajuste da umidade nos tubos percoladores, alterou-se o procedimento proposto por Stanford \& Smith (1972), pois a pressão de vácuo de $0,0789 \mathrm{MPa}$ (60 $\mathrm{cm}$ de $\mathrm{Hg}$ ) provocou compactação da mistura solo+areia e perda de argila dos tubos. O emprego de $0,0131 \mathrm{MPa}(10 \mathrm{~cm}$ de $\mathrm{Hg}$ ) solucionou os problemas, e a pressão empregada encontra-se no intervalo de pressão ótima, 0,0098 a $0,0296 \mathrm{MPa}(7,5$ a $22,5 \mathrm{~cm}$ de $\mathrm{Hg}$ ), definido por Wang et al. (2003) para o método de mineralização aeróbia com lixiviação.

Para o método de incubação anaeróbia de solo de curta duração, utilizou-se o procedimento descrito em Keeney \& Bremner (1966), que consiste na determinação do $\mathrm{N}^{-} \mathrm{NH}_{4}{ }^{+}$(Cantarella \& Trivelin, 2001) em suspensões de solo e água submetidas ou não à incubação a $40^{\circ} \mathrm{C}$, por sete dias. Amostras de $5 \mathrm{~g}$ de solo, em triplicata, foram transferidas para tubos de ensaio ( $15 \mathrm{~cm}$ de comprimento e $1,5 \mathrm{~cm}$ de diâmetro interno) contendo $12,5 \mathrm{~mL}$ de água deionizada, os quais foram tapados com filme de polietileno e mantidos em incubação em câmara tipo "BOD" a $40{ }^{\circ} \mathrm{C}$ por sete dias. Ao final do período de incubação, os tubos foram agitados por cerca de $15 \mathrm{~s}$, e as suspensões de solo foram transferidas para tubos de destilação com auxílio de $12,5 \mathrm{~mL}$ de solução de $\mathrm{KCl} 4 \mathrm{~mol} \mathrm{~L}^{-1}$, divididos em quatro alíquotas. $\mathrm{O} \mathrm{N}$ $\mathrm{NH}_{4}{ }^{+}$foi determinado nas suspensões empregando o método descrito em Cantarella \& Trivelin (2001). As quantidades de $\mathrm{N}^{-\mathrm{NH}_{4}}{ }^{+}$mineralizadas com o método de incubação anaeróbia $\left(\mathrm{N}_{\mathrm{an}}\right)$ foram obtidas por meio da diferença entre os teores de $\mathrm{N}-\mathrm{NH}_{4}{ }^{+}$determinados com e sem a incubação.

Com os mesmos solos, realizou-se experimento em casa de vegetação, em delineamento inteiramente ao acaso, com quatro repetições. Os vasos foram preparados a partir de anéis de PVC acoplados a "caps" (tampas de PVC para fechar a base dos anéis), cada um com capacidade para cerca de $12 \mathrm{~L}(24,5 \mathrm{~cm}$ de diâmetro x $25 \mathrm{~cm}$ de altura). Para evitar o escoamento preferencial da água, as paredes internas dos anéis de PVC foram lixadas, revestidas com resina líquida, e sobre ela foi aplicada mistura de areia grossa (diâmetro entre 1,5 mm e $4 \mathrm{~mm}$ ) e areia fina (diâmetro entre 0,5 e 1,5 mm), na proporção de 3:1 (v:v), lavada com solução de $\mathrm{HCl} 500 \mathrm{~mL} \mathrm{~L}^{-1}$ e água deionizada. $\mathrm{Na}$ parte inferior interna dos vasos, na região dos "caps", foi colocado sistema de drenagem constituído por mangueira de plástico com diâmetro de cerca de 0,79 e $15 \mathrm{~cm}$ de comprimento (3 $\mathrm{cm}$ para fora do vaso), bolinhas de isopor de $2,5 \mathrm{~cm}$ de diâmetro e mistura de areia lavada preenchendo os espaços.

Os vasos foram preenchidos com $10 \mathrm{dm}^{3}$ de solo previamente tratado com corretivos de acidez e adubos. Exceção feita aos solos 5, 9 e 10, os demais receberam mistura de $\mathrm{CaCO}_{3}$ p.a. e $4 \mathrm{MgCO}_{3} \cdot \mathrm{Mg}(\mathrm{OH})_{2} \cdot \mathrm{H}_{2} \mathrm{O}$ p.a., na proporção de Ca:Mg de 3:1, em quantidades para elevar o V a $70 \%$ e o teor de $\mathrm{Mg}^{2+}$ a um mínimo de $8 \mathrm{mmol}_{\mathrm{c}} \mathrm{dm}^{-3}$, conforme recomendações de Raij \& Cantarella (1996) para a cultura do milho. A adubação foi feita com 150, 100 e $50 \mathrm{mg} \mathrm{dm}^{-3}$ de $\mathrm{P}$, conforme classes de teores de $\mathrm{P}$ no solo para culturas anuais de $<15,16$ a 40 e $>40 \mathrm{mg} \mathrm{dm}^{-3}$, respectivamente (Raij et al., 1996); $20 \mathrm{mg} \mathrm{dm}^{-3} \mathrm{de} \mathrm{S}$; $0,25 \mathrm{mg} \mathrm{dm}^{-3}$ de B; $1,00 \mathrm{mg} \mathrm{dm}^{-3} \mathrm{de} \mathrm{Cu} ; 1,50 \mathrm{mg} \mathrm{dm}^{-3}$ de $\mathrm{Zn}$ e $0,02 \mathrm{mg} \mathrm{dm}^{-3}$ de Mo. Os adubos empregados foram $\mathrm{Ca}\left(\mathrm{H}_{2} \mathrm{PO}_{4}\right)_{2} \cdot \mathrm{H}_{2} \mathrm{O}, \mathrm{K}_{2} \mathrm{SO}_{4}, \mathrm{H}_{3} \mathrm{BO}_{3}, \mathrm{CuSO}_{4} \cdot 5 \mathrm{H}_{2} \mathrm{O}$, $\mathrm{ZnSO}_{4} \cdot 7 \mathrm{H}_{2} \mathrm{O}$ e $\left(\mathrm{NH}_{4}\right)_{6} \mathrm{Mo}_{7} \mathrm{O}_{24} \cdot 4 \mathrm{H}_{2} \mathrm{O}$, respectivamente. Nos solos 18 e 20 foram aplicados $2 \mathrm{mg} \mathrm{dm}^{-3} \mathrm{de} \mathrm{Mn}$ na forma de $\mathrm{MnCl}_{2} \cdot 2 \mathrm{H}_{2} \mathrm{O}$. Aplicou-se, também, $\mathrm{KCl}$ em quantidade para balancear o teor de $\mathrm{K}$ trocável dos solos a $6,4 \mathrm{mmol}_{\mathrm{c}} \mathrm{dm}^{-3}$, obtida após a adição de $\mathrm{K}_{2} \mathrm{SO}_{4}$.

O tempo de reação dos corretivos e adubos com os solos foi de 32 dias, período em que a umidade dos solos foi mantida a $60 \%$ da capacidade de retenção de água, com água deionizada.

Cinco plantas de milho (Agromen 2012, semeado em 18-03-2006) cresceram por 50 dias, em cada vaso, mantendo-se a umidade dos solos a $60 \%$ da capacidade de retenção de água por meio de pesagens e reposição da água perdida com água deionizada. Visando simular precipitação pluvial, foram feitas percolações com água deionizada aos 30 e 40 dias após a semeadura do milho, de modo a obter $250 \mathrm{~mL}$ de solução percolada por vaso, por aplicação. Um dia após cada percolação de água, foram feitas aplicações de $150 \mathrm{mg} \mathrm{dm}^{-3} \mathrm{de} \mathrm{K}$ e $20 \mathrm{mg} \mathrm{dm}^{-3}$ de $\mathrm{S}$ em todos os vasos, utilizando soluções contendo $\mathrm{KCl}$ e $\mathrm{K}_{2} \mathrm{SO}_{4}$. Na colheita, as plantas foram cortadas a $1 \mathrm{~cm}$ da superfície do solo. A parte aérea das plantas de milho foi lavada em solução de detergente a $1 \mathrm{~mL} \mathrm{~L}^{-1}$, em água deionizada, e seca em estufa com circulação forçada de ar a cerca de $65{ }^{\circ} \mathrm{C}$ até peso constante, para obter matéria seca da parte aérea (Bataglia et al., 1983). As amostras foram moídas em moinho tipo Wiley e analisadas quanto a N total conforme método descrito em Sarruge \& Haag (1974). 
A partir dos resultados de $\mathrm{N}_{\mathrm{mac}}$ em 30 semanas de incubação aeróbia, foram calculados os valores de $\mathrm{N}$ potencialmente mineralizável $\left(\mathrm{N}_{0}\right)$ de cada solo, por meio do modelo exponencial de primeira ordem ou de crescimento (Stanford \& Smith, 1972). Realizou-se ajuste dos resultados de $\mathrm{N}_{\mathrm{mac}}$ a modelos exponenciais duplos (Camargo et al., 2002) e de $\mathrm{N}_{\mathrm{m}}$ a modelo exponencial de decrescimento $\left(\mathrm{N}_{\mathrm{m}}=\mathrm{N}_{\mathrm{eq}}+\mathrm{Ae}^{-\mathrm{t} / \mathrm{k}}\right.$; em que $\mathrm{N}_{\mathrm{m}}=\mathrm{N}$ mineralizado no tempo $\mathrm{t}, \mathrm{N}_{\mathrm{eq}}=\mathrm{N}$ mineralizado no equilíbrio, $\mathrm{A}=$ amplitude $\mathrm{e} \mathrm{k}=$ constante de decréscimo). Foram também realizadas análises de correlação linear entre atributos do solo e as quantidades de $\mathrm{N}_{\text {mac }}$ obtidas nos períodos de avaliação da incubação aeróbia, e entre os resultados de $\mathrm{N}_{\text {mac }}, \mathrm{N}_{0}$ e de $\mathrm{N}_{\mathrm{an}}$ e a concentração e a quantidade acumulada de $\mathrm{N}$ na matéria seca da parte aérea das plantas de milho.

\section{RESULTADOS E DISCUSSÃO}

Para todos os solos, foi observado aumento abrupto de $\mathrm{N}$ mineral nos períodos iniciais da incubação, havendo subsequente diminuição e estabilização a partir da quarta semana (Figura 1a). Esta cinética de mineralização do Né típica em estudos de incubação de longa duração com amostras deformadas de solo, seja em condições aeróbias, seja em meio anaeróbio (Stanford \& Smith, 1972; Pöttker \& Tedesco, 1979; Camargo et al., 1997; Gonçalves et al., 2001; Wang et al., 2003; Rhoden et al., 2006). A maior quantidade de $\mathrm{N}$ mineralizada nos períodos iniciais é atribuída às frações orgânicas de fácil mineralização (Pöttker \& Tedesco, 1979; Wang et al., 2003; Rhoden et al., 2006), provenientes, possivelmente, da reciclagem da biomassa microbiana (Mengel, 1996), estimulada pela aeração resultante do preparo e manuseio das amostras de solo que induz aumento da atividade microbiana (Stanford \& Smith, 1972; Camargo et al., 1997; Rhoden et al., 2006). Resultados semelhantes foram obtidos por Cabrera \& Kissel (1988), os quais, ao compararem a mineralização do $\mathrm{N}$ orgânico em amostras deformadas e indeformadas de solo, não observaram quantidades iniciais elevadas de $\mathrm{N}_{\mathrm{m}}$ nestas últimas.

A partir da tendência obtida para os resultados de $\mathrm{N}_{\mathrm{m}}$ (aumento inicial com posterior diminuição e estabilização), em 20 (91\%) dos 22 solos a mineralização do $\mathrm{N}$ ajustou-se ao modelo exponencial simples de decrescimento, com coeficientes de determinação variando de $0,41^{*}$ a $0,99 * *$ (Quadro 2 ). Medindo a liberação de C- $\mathrm{CO}_{2}$ do solo em câmara portátil, Scala Junior et al. (2001) observaram que sistemas de preparo do solo com revolvimento intenso, com arado escarificador ou arado de disco seguido de grade niveladora, resultaram em mineralização intensa da matéria orgânica do solo (MOS) nos dias subsequentes às operações de preparo do solo, com posterior diminuição e estabilização do processo. Nesse caso, os autores observaram que as quantidades de C$\mathrm{CO}_{2}$ liberadas em 1, 2, 3, 4, 8,5 e 15,5 dias após as operações de preparo do solo ajustaram-se ao modelo exponencial de decrescimento $\left(R^{2}=0,97^{* *}\right.$ a $\left.0,99^{* *}\right)$. Assim, pode-se especular que a cinética de mineralização líquida de $\mathrm{N}$ em incubação aeróbia de amostras de solo deformadas, caracterizada por aumento abrupto e posterior estabilização do $\mathrm{N}_{\mathrm{m}}$, assemelha-se ao que ocorre em sistemas convencionais de preparo do solo, nos quais há aumento da aeração devido ao revolvimento e à exposição dos agregados à oxidação da MOS após arações e gradagens.

Em todos os solos, houve ajuste dos resultados de $\mathrm{N}_{\mathrm{mac}}$ à equação exponencial de primeira ordem ou de crescimento (Stanford \& Smith, 1972), com valores de $\mathrm{R}^{2}$ que variaram de $0,78^{* *}$ a $0,97^{* *}$ (Quadro 2). A partir dos resultados do quadro 2 , observou-se que o percentual de $\mathrm{N}_{0}$ em relação ao $\mathrm{N}$ total dos solos variou de 12,7 a $21,2 \%$, com média de $16,5 \%$, valor próximo à média real obtida $\left(\mathrm{N}_{\mathrm{mac}} / \mathrm{N}\right.$ total), que foi de $15,1 \%$ em média, considerando as 30 semanas de avaliação e descontando as quantidades de $\mathrm{N}_{\mathrm{m}}$ iniciais. Por meio da triplicata das densidades globais de cada solo nas amostras deformadas, determinou-se que as quantidades de $\mathrm{N}_{0}$ extrapoladas para a profundidade
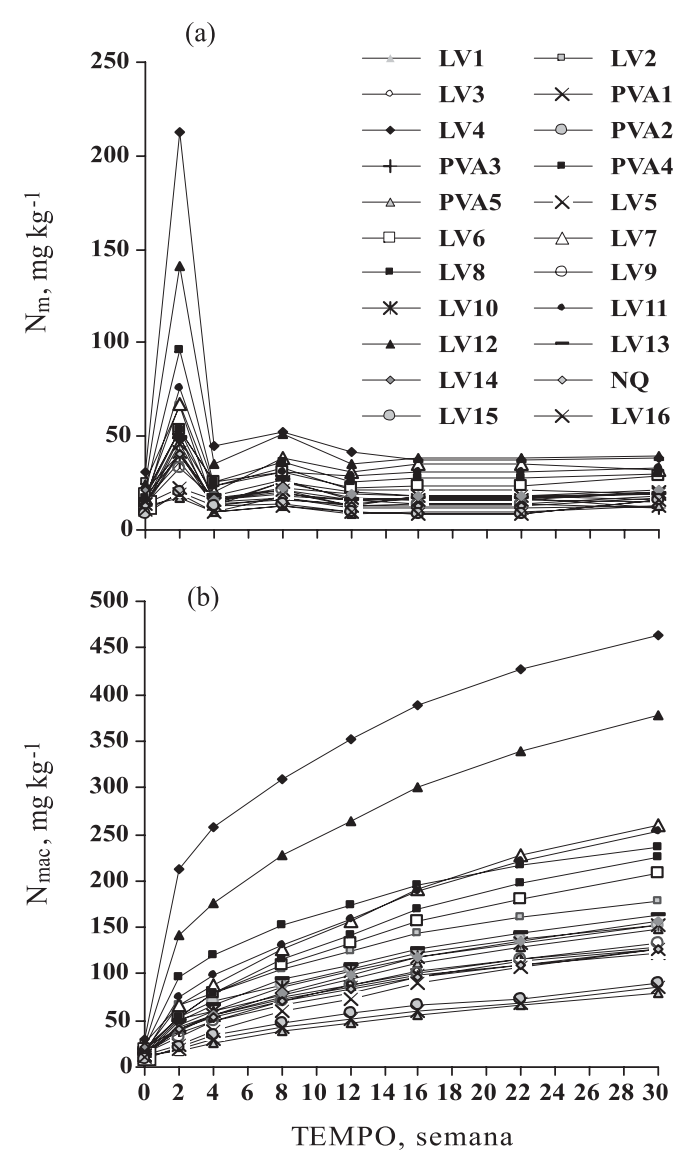

Figura 1. Nitrogênio mineralizado $\left(\mathrm{N}_{\mathrm{m}}\right)$ (a) e nitrogênio mineralizado acumulado $\left(\mathrm{N}_{\text {mac }}\right)(\mathrm{b})$ em ensaio de incubação aeróbia de longa duração. 
Quadro 2. Nitrogênio mineralizado em amostras de solos submetidas à incubação aeróbia e anaeróbia, estimativa de $\mathrm{N}$ potencialmente mineralizável, coeficientes de determinação dos modelos exponenciais, matéria seca, concentração de $\mathrm{N}$ e $\mathrm{N}$ acumulado na parte aérea de plantas de milho

\begin{tabular}{|c|c|c|c|c|c|c|c|c|}
\hline \multirow{2}{*}{ Solo ${ }^{(1)}$} & \multirow{2}{*}{$\mathrm{N}_{\text {mac }}{ }^{(2)}$} & \multirow{2}{*}{$\mathbf{N}_{0}{ }^{(3)}$} & \multicolumn{2}{|c|}{$\mathbf{R}^{2}$} & \multirow{2}{*}{$\mathrm{N}_{a n}^{\left({ }^{(6)}\right.}$} & \multirow{2}{*}{$\operatorname{MSPA}^{(7)}$} & \multirow{2}{*}{$\mathbf{N}_{\text {conc }}{ }^{(8)}$} & \multirow{2}{*}{$\mathbf{N}_{\text {acum }}{ }^{(9)}$} \\
\hline & & & MESD $^{(4)}$ & MESC $^{(5)}$ & & & & \\
\hline & \multicolumn{2}{|c|}{$\mathrm{mg} \mathrm{kg}^{-1}$} & & & $\mathrm{mg} \mathrm{kg}^{-1}$ & g/vaso & $\mathrm{g} \mathrm{kg}^{-1}$ & g/vaso \\
\hline $\mathrm{LV} 1$ & 149 & 143 & $0,95^{* *}$ & $0,88^{* *}$ & 21,1 & 85,11 & 8,64 & 0,74 \\
\hline $\mathrm{LV} 2$ & 178 & 180 & $0,96 * *$ & $0,89 * *$ & 12,0 & 87,50 & 8,87 & 0,77 \\
\hline $\mathrm{LV} 3$ & 129 & 131 & $0,93^{* *}$ & $0,89 * *$ & 23,6 & 46,30 & 8,68 & 0,40 \\
\hline PVA1 & 123 & 121 & $0,97 * *$ & $0,87^{* *}$ & 14,2 & 85,11 & 7,70 & 0,65 \\
\hline $\mathrm{LV} 4$ & 465 & 426 & $0,99 * *$ & $0,89 * *$ & 135,5 & 47,98 & 21,68 & 1,04 \\
\hline PVA2 & 152 & 164 & $0,99 * *$ & $0,94 * *$ & 21,6 & 104,11 & 7,69 & 0,80 \\
\hline PVA3 & 126 & 122 & $0,95^{* *}$ & $0,96 * *$ & 42,0 & 89,32 & 7,07 & 0,63 \\
\hline PVA4 & 237 & 221 & $0,98^{* *}$ & $0,91^{* *}$ & 54,0 & 137,07 & 8,69 & 1,19 \\
\hline PVA5 & 79 & 87 & $0,05 \mathrm{Ns}$ & $0,96 * *$ & 6,4 & 47,08 & 5,80 & 0,27 \\
\hline LV5 & 127 & 153 & $0,41^{*}$ & $0,78^{* *}$ & 14,8 & 38,99 & 6,88 & 0,27 \\
\hline $\mathrm{LV} 6$ & 209 & 231 & $0,92 * *$ & $0,89 * *$ & 52,2 & 72,37 & 9,30 & 0,67 \\
\hline LV7 & 259 & 310 & $0,86 * *$ & $0,94 * *$ & 50,5 & 68,72 & 13,14 & 0,90 \\
\hline LV8 & 226 & 271 & $0,85^{* *}$ & $0,86^{* *}$ & 18,3 & 49,77 & 11,34 & 0,56 \\
\hline LV9 & 134 & 152 & $0,82^{* *}$ & $0,97 * *$ & 7,9 & 55,58 & 7,23 & 0,40 \\
\hline LV10 & 153 & 162 & $0,87 * *$ & $0,98^{* *}$ & 31,6 & 79,92 & 7,32 & 0,58 \\
\hline LV11 & 254 & 289 & $0,97 * *$ & $0,94 * *$ & 48,4 & 67,08 & 9,60 & 0,64 \\
\hline $\mathrm{LV} 12$ & 378 & 374 & $0,98 * *$ & $0,96^{* *}$ & 51,7 & 80,46 & 17,15 & 1,37 \\
\hline LV13 & 164 & 163 & $0,92^{* *}$ & $0,97^{* *}$ & 4,2 & 49,20 & 11,34 & 0,56 \\
\hline LV14 & 157 & 169 & $0,93^{* *}$ & $0,96^{* *}$ & 2,3 & 89,44 & 7,57 & 0,68 \\
\hline NQ & 126 & 118 & $0,95^{* *}$ & $0,97^{* *}$ & 4,8 & 49,83 & 11,90 & 0,59 \\
\hline $\mathrm{LV} 15$ & 90 & 89 & $0,61^{*}$ & $0,94^{* *}$ & 15,6 & 53,88 & 5,81 & 0,31 \\
\hline LV16 & 85 & 93 & $0,01^{\mathrm{NS}}$ & $0,92^{* *}$ & 6,7 & 49,47 & 6,65 & 0,33 \\
\hline
\end{tabular}

(1) LV: Latossolo Vermelho; PVA: Argissolo Vermelho-Amarelo; NQ: Neossolo Quartzarênico. ${ }^{(2)} \mathrm{N}_{\text {mac }}$ : N mineralizado acumulado em 30 semanas de incubação aeróbia. ${ }^{\left({ }^{3)}\right.} \mathrm{N}_{0}: \mathrm{N}$ potencialmente mineralizável. ${ }^{(4)}$ Modelo exponencial simples de decrescimento. ${ }^{(5)}$ Modelo exponencial simples de crescimento. ${ }^{(6)} \mathrm{N}_{\mathrm{an}}: \mathrm{N}$ mineralizado em incubação anaeróbia. ${ }^{(7)}$ MSPA: matéria seca da parte aérea das plantas de milho. ${ }^{(8)} \mathrm{N}_{\text {conc }}$ : concentração de $\mathrm{N}$ na MSPA. ${ }^{(9)} \mathrm{N}_{\text {acum }}$ : acúmulo de $\mathrm{N}$ na MSPA. **, * e ${ }^{\text {NS: }}$ : significativo a 1 e a $5 \%$, e não-significativo, respectivamente.

de 0 a $20 \mathrm{~cm}$ de um hectare seriam de 240 (PVA4) a 959 (LV4) kg ha-1 de N, com média de $479 \mathrm{~kg} \mathrm{ha}^{-1}$ de $\mathrm{N}$, o que sugere, aparentemente, que tais quantidades seriam muito maiores do que as quantidades de $\mathrm{N}$ orgânico que seriam mineralizadas nas condições naturais destes solos.

Foram observadas correlações significativas entre os teores de $\mathrm{N}_{\text {mac }}$ e os de MOS e de $\mathrm{N}$ total do solo, com coeficientes de correlação (R) maiores entre $\mathrm{N}_{\text {mac }}$ e $\mathrm{N}$ total do solo (Quadro 3), o que indica que os teores de $\mathrm{N}$ total do solo são índices mais confiáveis do que os de MOS para predizer a mineralização líquida do $\mathrm{N}$ orgânico. Por meio da correlação entre os teores de MOS e de $\mathrm{N}$ total do solo, verificou-se que estes atributos explicam 62 e $86 \%$ da variação das quantidades de $\mathrm{N}_{\text {mac }}$, respectivamente. Na predição da mineralização líquida do N orgânico em solos do Rio Grande do Sul, Pöttker \& Tedesco (1979) e Gianello \& Bremner (1986) observaram superioridade dos teores de $\mathrm{N}$ total em relação aos de MOS, enquanto que Camargo et al. (1997) e Rhoden et al. (2006) observaram superioridade da MOS em relação ao N total do solo.

Os valores de $R$ para as correlações de $\mathrm{N}_{\text {mac }}$ com MOS e com $\mathrm{N}$ total do solo aumentaram no decorrer das avaliações do método de incubação de solo, de $0,54^{* *}$ a $0,79^{* *}$ e de $0,61^{* *}$ a $0,93^{* *}$, respectivamente (Quadro 3), o que sugere que tais índices podem predizer a mineralização líquida do $\mathrm{N}$ orgânico em longo prazo. Nos intervalos de 0, 2, 4, 8, 12, 16, $22 \mathrm{e}$ 30 semanas, os percentuais de $\mathrm{N}_{\text {mac }}$ em relação ao $\mathrm{N}$ total do solo foram de 1,4, 4,8, 6,3, 8,5, 10,0, 11,7, 13,3 e 15,1\%, respectivamente. Assim, aumentou a participação do $\mathrm{N}_{\mathrm{mac}}$ em relação ao $\mathrm{N}$ total do solo, estreitando as correlações entre os teores de $\mathrm{N}$ total e de MOS com o $\mathrm{N}_{\text {mac }}$ ao longo do tempo (Quadro 3). Como os valores de $\mathrm{N}_{0}$ foram calculados a partir dos resultados de $\mathrm{N}_{\text {mac }}$ em 30 semanas de incubação aeróbia, as correlações entre MOS (Quadro 1) $x \mathrm{~N}_{0}$ (Quadro 2) e $\mathrm{N}$ total de solo (Quadro 1) $x \mathrm{~N}_{0}$ também foram significativas $\left(\mathrm{N}_{0}=13,016+5,03 \mathrm{MOS} ; \mathrm{R}=\right.$ $0,86^{* *}$ e $\left.\mathrm{N}_{0}=33,195+129,47 \mathrm{~N}_{\text {total }} ; \mathrm{R}=0,97 * *\right)$. 
Quadro 3. Coeficientes de correlação $(R)$ entre as quantidades de $N$ mineralizadas acumuladas $\left(N_{\text {mac }}\right)$ e atributos de solo (MOS, $N$ total, areia, silte e argila) e planta (MSPA, $\mathbf{N}_{\text {conc }}$ e $\mathrm{N}_{\text {acum }}$ ) durante 30 semanas de avaliação

\begin{tabular}{|c|c|c|c|c|c|c|c|c|}
\hline \multirow{2}{*}{ Variável $^{(1)}$} & \multicolumn{8}{|c|}{$\mathbf{N}_{\text {mac }}$ ao longo do tempo (semana) } \\
\hline & 0 & 2 & 4 & 8 & 12 & 16 & 22 & 30 \\
\hline & \multicolumn{8}{|c|}{$\mathrm{R}^{(2)}$} \\
\hline MOS & $0,54^{* *}$ & $0,64^{* *}$ & $0,66^{* *}$ & $0,70^{* *}$ & $0,73^{* *}$ & $0,75^{* *}$ & $0,77^{* *}$ & $0,79^{* *}$ \\
\hline $\mathrm{N}$ total & $0,61^{* *}$ & $0,80^{* *}$ & $0,83^{* *}$ & $0,86^{* *}$ & $0,88^{* *}$ & $0,90 * *$ & $0,91^{* *}$ & $0,93 * *$ \\
\hline Areia & $-0,40 \mathrm{NS}$ & $-0,50^{*}$ & $-0,54^{* *}$ & $-0,59^{* *}$ & $-0,62^{* *}$ & $-0,66^{* *}$ & $-0,68^{* *}$ & $-0,71^{* *}$ \\
\hline Silte & $0,30 \mathrm{NS}$ & $0,45^{*}$ & $0,48^{*}$ & $0,53^{* *}$ & $0,56^{* *}$ & $0,59 * *$ & $0,61^{* *}$ & $0,64 * *$ \\
\hline Argila & $0,42^{*}$ & $0,48^{*}$ & $0,51^{*}$ & $0,56^{* *}$ & $0,59 * *$ & $0,62^{* *}$ & $0,64^{* *}$ & $0,66^{* *}$ \\
\hline MSPA & $0,34 \mathrm{NS}$ & $0,16 \mathrm{NS}$ & $0,14 \mathrm{NS}$ & $0,13 \mathrm{NS}$ & 0,13 NS & $0,12 \mathrm{NS}$ & 0,12 NS & $0,11 \mathrm{NS}$ \\
\hline $\mathrm{N}_{\text {conc. }}$ & $0,67^{* *}$ & $0,90^{* *}$ & $0,90 * *$ & $0,91^{* *}$ & $0,91^{* *}$ & $0,91^{* *}$ & $0,91^{* *}$ & 0,91 ** \\
\hline $\mathrm{N}_{\text {acum. }}$ & $0,74 * *$ & $0,79^{* *}$ & $0,66^{* *}$ & $0,75^{* *}$ & $0,75^{* *}$ & $0,73^{* *}$ & $0,73^{* *}$ & $0,59 * *$ \\
\hline
\end{tabular}

${ }^{(1)}$ MOS: matéria orgânica do solo; $\mathrm{N}$ total: $\mathrm{N}$ total do solo; MSPA: matéria seca da parte aérea das plantas de milho; $\mathrm{N}_{\text {conc }}$ : concentração de $\mathrm{N}$ na MSPA; $\mathrm{N}_{\text {acum }}$ : acúmulo de $\mathrm{N}$ na MSPA. ${ }^{(2) \mathrm{NS}}$, ** $\mathrm{e}^{*}$ : não-significativo e significativo a 1 e a $5 \%$, respectivamente.

A partir dos resultados de $\mathrm{N}_{\mathrm{m}}$ (Figura 1a), foram calculadas as taxas de mineralização de $\mathrm{N}$ orgânico em cada período de avaliação, dividindo a quantidade de $\mathrm{N}_{\mathrm{m}}$ pelo respectivo período de tempo (em semanas). Os resultados médios para os 22 solos nas $2,4,8,12$, 16, 22 e 30 semanas de incubação foram de $29,9,6$, $5,5,4$ e $3 \mathrm{mg} \mathrm{kg}^{-1}$ por semana, respectivamente. A temperatura e a umidade constantes durante a incubação aeróbia provavelmente favoreceram a liberação intensa e gradativa de $\mathrm{N}$ inorgânico das frações orgânicas mais lábeis, seguidas da mineralização constante das frações de maior recalcitrância. No entanto, os resultados de $\mathrm{N}_{\mathrm{mac}}$ ao longo da incubação aeróbia dos solos não se ajustaram aos modelos exponenciais duplos (Camargo et al., 2002), os quais pressupõem que existem duas frações de $\mathrm{N}$ orgânico que possuem taxas distintas de mineralização. Esse resultado foi atribuído à ausência de estabilização das quantidades de $\mathrm{N}_{\mathrm{mac}}$ no decorrer das avaliações, o que pode ser constatado pelas quantidades de $\mathrm{N}_{\mathrm{m}}$ constantes observadas em todos os solos a partir, principalmente, da $12^{a}$ semana de avaliação (Figuras 1a, b).

As correlações entre $N_{\text {mac }}$ e os teores de areia foram negativas e significativas a partir da $2^{\mathrm{a}}$ semana de incubação, enquanto as correlações entre $\mathrm{N}_{\mathrm{mac}} \mathrm{e}$ os teores de argila e silte foram positivas e significativas a partir do início (tempo 0) e da segunda semana de incubação, respectivamente. Em todos os casos, os valores de $\mathrm{R}$ aumentaram no decorrer das avaliações (Quadro 3). A correlação positiva entre as quantidades de $\mathrm{N}_{\mathrm{mac}}$ e as frações texturais mais finas $(\phi<0,02 \mathrm{~mm})$ pode ser atribuída à maior quantidade de compostos nitrogenados em complexos organominerais estáveis, que liberariam $\mathrm{N}$ inorgânico proporcionalmente para a solução do solo. Em solos de clima temperado, Mengel (1996) relata que argilas protegem proteínas e peptídeos da ação enzimática microbiana, estocando N orgânico que pode contribuir para o aumento do potencial de mineralização dos solos. Por outro lado, o aumento dos valores de $R$ entre $\mathrm{N}_{\mathrm{mac}}$ e os teores de areia ao longo do tempo $\left(-0,50^{*}\right.$ a $\left.-0,71^{* *}\right)$ pode ter sido consequência indireta da correlação negativa $\left(R=-0,85^{* *}\right)$ entre MOS e areia das amostras. Assim, quanto maior o teor de areia do solo, menor o teor de MOS e menor a fração do N orgânico suscetível à mineralização ao longo do tempo.

As quantidades de $\mathrm{N}_{\mathrm{mac}}$ correlacionaram-se significativamente em todos os períodos de avaliação com a concentração $\left(\mathrm{N}_{\text {conc }}\right)$ e o acúmulo de $\mathrm{N}\left(\mathrm{N}_{\text {acum }}\right)$ na matéria seca da parte aérea (MSPA) das plantas de milho no final do experimento, com valores de $R$ variando de $0,67^{* *}$ a $0,91^{* *}$ e de $0,59^{* *}$ a $0,79^{* *}$, respectivamente. Não houve correlações significativas entre as quantidades $\mathrm{N}_{\mathrm{mac}}$ e as produções de MSPA (Quadro 3). A ausência de correlação com a MSPA deve ser em parte associada ao solo LV4, coletado em área de mata, rico em MOS e com mineralização líquida de $\mathrm{N}$ elevada, mas com baixa produção de MSPA, semelhante aos solos com baixa mineralização líquida de $\mathrm{N}$ orgânico. Também nesse caso, como $\mathrm{N}_{0}$ foi calculado a partir de $\mathrm{N}_{\text {mac }}$, houve correlação entre $\mathrm{N}_{0}$ e $\mathrm{N}_{\text {conc }}$ ou $\mathrm{N}_{\text {acum }}\left(\mathrm{N}_{0}=-10,943+20,933 \mathrm{~N}_{\text {conc }}, \mathrm{R}=\right.$ $\left.0,86^{* *} ; \mathrm{N}_{0}=-35,875+235,43 \mathrm{~N}_{\text {acum }} ; \mathrm{R}=0,73^{* *}\right)$. Esses resultados estão de acordo com os obtidos por Camargo et al. (1997) e Rhoden et al. (2006), que observaram que os valores de $\mathrm{N}_{0}$ caracterizam adequadamente a disponibilidade de $\mathrm{N}$ para o milho e o arroz inundado, respectivamente. Os valores de $R$ entre $N_{\text {conc }}$ e $N_{\text {mac }}$ foram maiores do que os valores de $R$ entre $\mathrm{N}_{\text {conc }}$ e $\mathrm{N}_{0}$ a partir da $2^{\mathrm{a}}$ semana de incubação, em sete avaliações, e os valores de $R$ entre $N_{\text {acum }}$ e $N_{\text {mac }}$ foram maiores que os de $\mathrm{N}_{\text {acum }}$ e $\mathrm{N}_{0}$ em quatro avaliações (tempo 0 e $2^{\mathrm{a}}, 8^{\mathrm{a}}$ e $12^{\mathrm{a}}$ semanas), iguais em duas avaliações $\left(16^{\mathrm{a}}\right.$ e $22^{\mathrm{a}}$ semanas) e menores em duas avaliações $\left(4^{\mathrm{a}}\right.$ e $30^{\mathrm{a}}$ semanas). Assim, a maior parte das correlações 
envolvendo os valores reais obtidos $\left(\mathrm{N}_{\text {mac }}\right)$ foi mais estreita do que as correlações que envolveram os valores obtidos a partir de modelagem matemática $\left(\mathrm{N}_{0}\right)$ dos resultados.

Considerando que neste trabalho nem todo $\mathrm{N}$ mineralizado tornou-se disponível às plantas de milho devido às lixiviações de $\mathrm{N}$, à imobilização microbiana, e, possivelmente, às perdas de $\mathrm{N}$ por desnitrificação, o método de incubação aeróbia de solo de longa duração pode ser utilizado como índice de mineralização e disponibilidade de $\mathrm{N}$ do solo, não requerendo, necessariamente, período de incubação de 30 semanas de avaliação seguido de modelagem matemática dos resultados. Os valores de $\mathrm{R}$ obtidos entre $\mathrm{N}_{\text {mac }}$ determinado no método de incubação e $\mathrm{N}_{\text {conc }}$ e $\mathrm{N}_{\text {acum }}$ nas plantas de milho foram maiores no intervalo de 2 a 8 semanas de incubação aeróbia (Quadro 3). Desse resultado há implicações práticas como maior facilidade para obtenção dos resultados, simplicidade e economia de tempo, e reagentes para as avaliações (lixiviações de N, aplicações de solução nutritiva sem $\mathrm{N}$ e ajustes de umidade nos tubos de percolação) e análises químicas de $\mathrm{N}$ inorgânico.

As quantidades de $\mathrm{N}$ obtidas com o método de incubação anaeróbia de curta duração $\left(\mathrm{N}_{\mathrm{an}}\right)$ variaram de 2,3 (LV14) a 135,5 mg kg-1 (LV4), com média de $29,1 \mathrm{mg} \mathrm{kg}^{-1}$ (Quadro 2). As correlações entre as quantidades de $\mathrm{N}-\mathrm{NH}_{4}{ }^{+}$mineralizadas no método de incubação anaeróbia e os teores de MOS e de N total do solo foram significativas a $1 \%$, com valores de $R$ iguais a 0,62 e 0,79, respectivamente. Para as correlações com essas variáveis, Gianello \& Bremner (1986) observaram valores de $\mathrm{R}$ iguais a 0,75 e 0,79 (0,1\%), respectivamente, em 33 solos do Rio Grande do Sul, enquanto Cantarella et al. (1994) não observaram significância para estas correlações em 20 solos do Estado de São Paulo.

Os resultados obtidos com o método de incubação anaeróbia de curta duração correlacionaram-se significativamente com os valores de $\mathrm{N}_{\text {mac }}\left(\mathrm{R}=0,85^{* *}\right)$ e de $\mathrm{N}_{0}\left(\mathrm{R}=0,79^{* *}\right)$, obtidos com o método de incubação aeróbia de longa duração (Figura 2a,b), e com os valores de $\mathrm{N}_{\text {conc }}\left(\mathrm{R}=0,73^{* *}\right)$ e de $\mathrm{N}_{\text {acum }}\left(\mathrm{R}=0,61^{* *}\right)$ obtidos no experimento em casa de vegetação (Figura 2c,d). Aparentemente, um dado isolado dos demais (referente ao solo LV4) nos gráficos de correlação poderia ter resultado em valores de $R$ maiores, porém a exclusão deste ponto não causou diminuição das correlações entre os resultados do método de incubação anaeróbia de curta duração e os
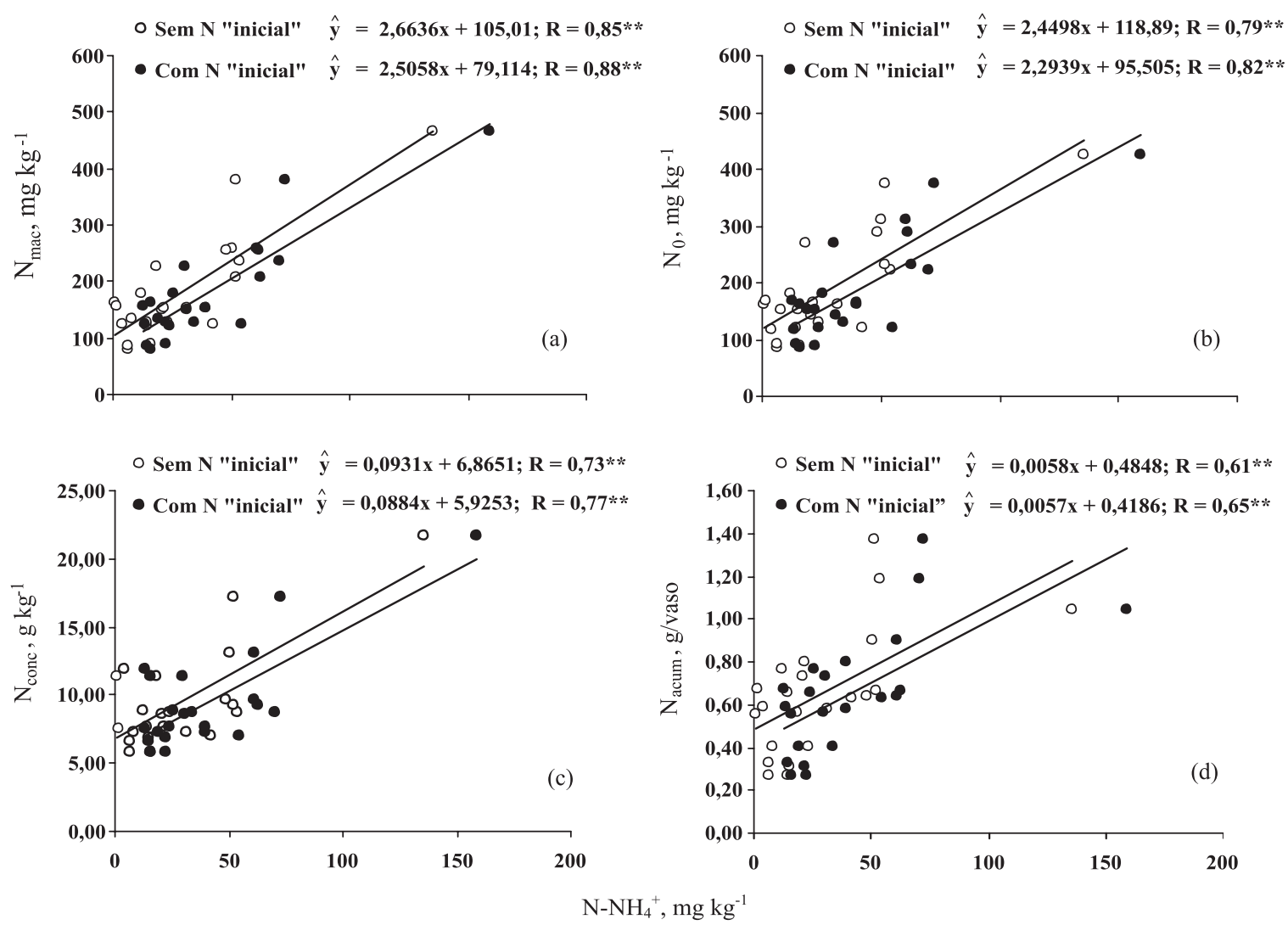

Figura 2. Correlações entre quantidades de $\mathrm{N}-\mathrm{NH}_{4}^{+}$obtidas após incubação anaeróbia por sete dias e $\mathrm{N}$ mineralizado acumulado em 30 semanas de incubação aeróbia $\left(N_{\text {mac }}\right)(a), N$ potencialmente mineralizável $\left(\mathrm{N}_{0}\right)(b)$, concentração $\left(\mathrm{N}_{\text {conc }}\right)(\mathrm{c})$ e acúmulo de $\mathrm{N}(\mathrm{N}$ ) (d) na matéria seca da parte aérea das plantas de milho. **: significativo a $1 \%$. 
obtidos com a incubação aeróbia de longa duração e com o $\mathrm{N}_{\text {conc }}$ e o $\mathrm{N}_{\text {acum. }}$. Keeney \& Bremner (1966), ao adaptarem o método de incubação anaeróbia que foi utilizado neste trabalho, observaram correlação significativa $\left(R=0,76^{* * *}\right)$ entre as quantidades de $\mathrm{N}-\mathrm{NH}_{4}{ }^{+}$mineralizadas e o $\mathrm{N}$ absorvido por plantas de azevém. Da mesma forma, Cantarella et al. (1994) observaram correlação $\left(\mathrm{R}=0,73^{* *}\right)$ entre o $\mathrm{N}^{-\mathrm{NH}_{4}}{ }^{+}$ mineralizado e as quantidades de N na MSPA de plantas de milho em 20 solos do Estado de São Paulo.

Considerando que o N- $\mathrm{NH}_{4}{ }^{+}$"inicial" determinado nas suspensões de solo com solução de $\mathrm{KCl} 4 \mathrm{~mol} \mathrm{~L}^{-1} \mathrm{e}$ $0,2 \mathrm{~g}$ de $\mathrm{MgO}$ poderia representar melhor a mineralização e a disponibilidade de $\mathrm{N}$ dos solos para as plantas, foram avaliadas as correlações entre as quantidades de $\mathrm{N}-\mathrm{NH}_{4}{ }^{+}$mineralizadas no ensaio de incubação anaeróbia de curta duração, incluindo o N$\mathrm{NH}_{4}{ }^{+}$"inicial", e os valores de $\mathrm{N}_{\text {mac }}$ e de $\mathrm{N}_{0}$, obtidos na incubação aeróbia de longa duração (Figura 2a,b), e de $\mathrm{N}_{\text {conc }}$ e de $\mathrm{N}_{\text {acum }}$, obtidos no experimento em casa de vegetação (Figura 2c,d). Foi verificado em todos os casos que, quando não se subtraiu o $\mathrm{N}-\mathrm{NH}_{4}{ }^{+}$"inicial" das quantidades de $\mathrm{N}-\mathrm{NH}_{4}{ }^{+}$mineralizadas, avaliadas após incubação anaeróbia, as correlações com os valores de $\mathrm{N}_{\text {mac }}, \mathrm{N}_{0}, \mathrm{~N}_{\text {conc }}$ e $\mathrm{N}_{\text {acum }}$ aumentaram. Admitindo que a secagem ao ar não seja um procedimento eficiente para preservar os teores de $\mathrm{N}$ $\mathrm{NH}_{4}{ }^{+}$em amostras de solo (Mattos Junior et al., 1995), pode-se supor que os teores de $\mathrm{N}-\mathrm{NH}_{4}{ }^{+}$"inicial" determinados neste trabalho sejam provenientes predominantemente da hidrólise alcalina da MOS (Sahrawat \& Ponnamperuma, 1978), e que contribuiriam para as formas de $\mathrm{N}$ orgânico mineralizáveis. Assim, a contabilização dos teores de $\mathrm{N}-\mathrm{NH}_{4}{ }^{+}$"inicial” para a mineralização anaeróbia, os quais representaram 14,8 (LV4) a 96,6\% (LV13) (média de $40,7 \%$ ) das quantidades de $\mathrm{N}-\mathrm{NH}_{4}{ }^{+}$ mineralizadas (Quadro 2), agregou maior precisão ao procedimento de incubação anaeróbia de solo, cujo CV passou de 14,2 para 7,4 \%.

Por meio da equação apresentada na figura $2 \mathrm{~b}\left(\mathrm{~N}_{0}\right.$ $\left.=2,2939 \mathrm{~N}_{\mathrm{an}}+95,505, \mathrm{R}=0,82^{* *}\right)$, podem-se estimar os valores de $\mathrm{N}_{0}$ a partir dos resultados obtidos com o método de incubação anaeróbia de solo de curta duração. Aplicando cinco doses de lodos de esgoto, provenientes de municípios do Estado de São Paulo (Franca e Barueri), em um Latossolo Vermelho de textura argilosa, Boeira \& Maximiliano (2004) também observaram correlação $\left(R=0,92^{* *}\right)$ entre o $\mathrm{N}-\mathrm{NH}_{4}{ }^{+}$mineralizado avaliado em incubação anaeróbia por sete dias e o $\mathrm{N}_{\mathrm{mac}}$ obtido com 105 dias de incubação aeróbia de solo, sem lixiviação.

\section{CONCLUSÕES}

1. As quantidades de N potencialmente mineralizável obtidas com a incubação aeróbia refletiram a mineralização e a disponibilidade de $\mathrm{N}$ em longo prazo.
2. O $\mathrm{N}$ total do solo foi melhor índice da mineralização potencial e líquida de $\mathrm{N}$ orgânico do que a matéria orgânica do solo, principalmente em longo prazo.

3. A quantidade líquida acumulada de $\mathrm{N}$ mineralizado em incubação aeróbia de longa duração representou melhor a disponibilidade de $\mathrm{N}$ para as plantas do que o $\mathrm{N}$ potencialmente mineralizável.

4. A incubação anaeróbia de curta duração pode ser utilizada para o cálculo da estimativa do $\mathrm{N}$ potencialmente mineralizável do solo.

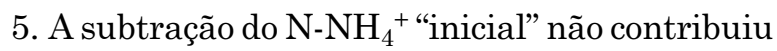
para melhorar a estimativa da mineralização e disponibilidade de $\mathrm{N}$ por meio de incubação anaeróbia.

\section{AGRADECIMENTO}

À FAPESP, pela concessão de bolsa de estudos ao primeiro autor (Processo 04/14.230-9) e de auxílio à pesquisa ao segundo autor (Processo 04/14.404-7).

\section{LITERATURA CITADA}

BATAGLIA, O.C.; FURLANI, A.M.C.; TEIXEIRA, J.P.F.; RAIJ, B.van \& QUAGGIO, J.A. Métodos de análise química de plantas. Campinas, Instituto Agronômico de Campinas, 1983. 48p. (Boletim técnico, 78)

BOEIRA, R.C. \& MAXIMILIANO, V.C.B. Determinação da fração de mineralização de nitrogênio de lodos de esgoto: Um método alternativo. Jaguariúna, Embrapa Meio Ambiente, 2004. 3p. (Comunicado Técnico, 13)

CABRERA, M.L. \& KISSEL, D.E. Potentially mineralizable nitrogen in disturbed and undisturbed soil samples. Soil Sci. Soc. Am. J., 52:1010-1015, 1988.

CAMARGO, F.A.O.; GIANELLO, C. \& VIDOR, C. Potencial de mineralização do nitrogênio em solos do Rio Grande do Sul. R. Bras. Ci. Solo, 21:575-579, 1997.

CAMARGO, F.A.O.; GIANELLO, C.; TEDESCO, M.J.; RIBOLDI, J.; MEURER, E.J. \& BISSANI, C.A. Modelos empíricos para a predição da mineralização do nitrogênio do solo. Ci. Rural, 32:393-399, 2002.

CAMARGO, O.A.; MONIZ, A.C.; JORGE, J.A. \& VALADARES, J.M.A.S. Métodos de análise química, mineralógica e física de solo do Instituto Agronômico de Campinas. Campinas, Instituto Agronômico de Campinas, 1986. 94p. (Boletim Técnico, 106)

CANTARELlA, H.; MATTOS Jr., D. \& RAIJ, B.van. Lime effect on soil $\mathrm{N}$ availability indexes as measured by plant uptake. Comm. Soil Sci. Plant Anal., 25:989-1006, 1994.

CANTARELlA, H. \& TRIVELIN, P.C.O. Determinação de nitrogênio inorgânico em solo pelo método da destilação a vapor. In: RAIJ, B.van; ANDRADE, J.C.; CANTARELLA, H. \& QUAGGIO, J.A., eds. Análise química para avaliação da fertilidade de solos tropicais. Campinas, Instituto Agronômico de Campinas, 2001. p.270-276. 
CURTIN, D. \& WEN, G. Organic matter fractions contributing to soil nitrogen mineralization potential. Soil Sci. Soc. Am. J., 63:410-415, 1999.

EMPRESA BRASILEIRA DE PESQUISA AGROPECUÁRIA EMBRAPA. Centro Nacional de Pesquisa de Solos. Sistema brasileiro de classificação de solos. Rio de Janeiro, 1999. $412 p$

GIANELLO, C. \& BREMNER, J.M. Comparison of chemical assessing potentially available organic nitrogen in soil. Comm. Soil Sci. Plant Anal., 17:215-236, 1986.

GONÇALVES, J.L.M.; MENDES, K.C.F.S. \& SASAKI, C.M Mineralização de nitrogênio em ecossistemas florestais naturais e implantados do Estado de São Paulo. R. Bras. Ci. Solo, 25:601-616, 2001.

KEENEY, D.R. \& BREMNER, J.M. Comparison and evaluation of laboratory methods of obtaining an index of soil nitrogen availability. Agron. J., 58:498-503, 1966.

MATTOS JUNIOR, D.; CANTARELLA, H. \& RAIJ, B.van. Manuseio e conservação de amostras de solo para preservação do nitrogênio inorgânico. R. Bras. Ci. Solo, 19:423-431, 1995.

MENGEL, K. Turnover of organic nitrogen in soil and its availability to crops. Plant Soil, 181:83-93, 1996

PÖTTKER, D. \& TEDESCO, M.J. Efeito do tipo e tempo de incubação sobre a mineralização da matéria orgânica e nitrogênio total em solos do Rio Grande do Sul. R. Bras. Ci. Solo, 3:20-24, 1979.

RAIJ, B.van; ANDRADE, J.C.; CANTARELLA, H. \& QUAGGIO, J.A. Análise química para avaliação da fertilidade de solos tropicais. Campinas, Instituto Agronômico de Campinas, 2001. 285p.

RAIJ, B.van \& CANTARELLA, H. Milho para grãos e silagem. In: RAIJ, B.van; CANTARELLA, H.; QUAGGIO, J.A. \& FURLANI, A.M.C., eds. Recomendações de adubação e calagem para o Estado de São Paulo. 2.ed. Campinas, Instituto Agronômico de Campinas, Fundação IAC, 1996. p.56-59.
RAIJ, B.van; CANTARELLA, H.; QUAGGIO, J.A. \& ABREU, C.A. Interpretação de resultados de análise de solo. In: RAIJ, B.van; CANTARELLA, H.; QUAGGIO, J.A. \& FURLANI, A.M.C., eds. Recomendações de adubação e calagem para o Estado de São Paulo. 2.ed. Campinas, Instituto Agronômico de Campinas, 1996. p.8-13. (Boletim Técnico, 100)

RHODEN, A.C.; SILVA, L.S.; CAMARGO, F.A.O.; BRITZKE, D. \& BENEDETTI, E.L. Mineralização anaeróbia do nitrogênio em solos de várzea do Rio Grande do Sul. Ci. Rural, 36:1780-1787, 2006.

SAHRAWAT, K.L. \& PONNAMPERUMA, F.N. Measurement of exchangeable $\mathrm{NH}_{4}{ }^{+}$in tropical rice soils. Soil Sci. Soc. Am. J., 42:282-283, 1978.

SARRUGE, J.R. \& HAAG, H.P. Análises químicas em plantas. Piracicaba, Escola Superior de Agricultura Luiz de Queiroz, 1974. 56p.

SCALA JUNIOR, N.; LOPES, A.; MARQUES JUNIOR, J. \& PEREIRA, G.T. Emissão de $\mathrm{CO}_{2}$ do solo em função dos sistemas de preparo. Eng. Agric., 21:270-275, 2001.

STANFORD, G. \& SMITH, S.J. Nitrogen mineralization potential of soils. Soil Sci. Soc. Am. J., 36:465-472, 1972.

TEDESCO, M.J.; VOLKWEISS, S.J. \& BOHNEN, H. Análises de solo, plantas e outros materiais. Porto Alegre, Universidade Federal do Rio Grande do Sul, 1985. $188 \mathrm{p}$.

WANG, W.J.; SMITH, C.J. \& CHEN, D. Towards a standardized procedure for determining the potentially mineralizable nitrogen of soil. Biol. Fert. Soils, 37:362-374, 2003.

WARING, S.A. \& BREMNER, J.M. Ammonium production in soil under waterlogged conditions as an index of nitrogen availability. Nature, 201:951-952, 1964.

ZECH, W.; SENESI, N.; GUGGEMBERGER, G.; KAISER, K.; LEHMANN, J.; MIANO, T.M.; MILTNER, A. \& SCHROTH, G. Factors controlling humification and mineralization of soil organic matter in the tropics. Geoderma, 79:117-161, 1997. 